Research Article

\title{
Circulating Tumor Cell Is a Clinical Indicator of Pretransplant Radiofrequency Ablation for Patients with Hepatocellular Carcinoma
}

\author{
Zhitao Chen (D), Tielong Wang, Chuanbao Chen, Xitao Hong, Jia Yu, Yihao Ma, Yiwen Guo, \\ Changjun Huang, Xiaoshun He $\mathbb{D}$, Weiqiang Ju $\mathbb{D}$, and Maogen Chen $\mathbb{D}$
}

\author{
Organ Transplant Center, First Affiliated Hospital of Sun Yat-sen University, \\ Guangdong Provincial Key Laboratory of Organ Donation and Transplant Immunology, \\ Guangdong Provincial International Cooperation Base of Science and Technology (Organ Transplantation), \\ Guangzhou 510080, China
}

Correspondence should be addressed to Xiaoshun He; gdtrc@163.com, Weiqiang Ju; weiqiangju@163.com, and Maogen Chen; chenmg3@mail.sysu.edu.cn

Received 15 July 2021; Revised 7 September 2021; Accepted 24 September 2021; Published 19 October 2021

Academic Editor: Irena Ilic

Copyright (C) 2021 Zhitao Chen et al. This is an open access article distributed under the Creative Commons Attribution License, which permits unrestricted use, distribution, and reproduction in any medium, provided the original work is properly cited.

\begin{abstract}
Introduction. It is of great significance to confirm reliable indicators for the guidance of pretransplant radiofrequency ablation (RFA) for hepatocellular carcinoma (HCC). In this study, we aim to investigate whether circulating tumor cell (CTC) status is a clinical indicator for RFA before liver transplantation (LT) in HCC patients. Method. CTC analyses were measured in 79 HCC patients. Clinical outcomes including progression-free (PFS) and overall survival (OS) were compared and analyzed between patients with and without pretransplant RFA. Result. Forty-two patients were detected as CTC-positive and 18 patients received pretransplant RFA. Recurrence was correlated with CTC count $(P=0.024)$, tumor number $(P=0.035)$, liver cirrhosis $(P=0.001)$, Milan criteria $(P=0.003)$, and University of California San Francisco (UCSF) criteria $(P=0.001)$. Kaplan-Meier analysis revealed that patients with CTC-positive had a lower PFS rate $(P=0.0257)$. For CTC-positive patients, the PFS rate of the pretransplant RFA group was significantly higher than the non-pretransplant RFA group (100\% vs. 46.7\%, $P=0.0236)$. For CTCnegative patients, both PFS rate and OS rate were similar and without significant differences. In multivariate analysis, pretransplant RFA was the independent factor for PFS $(P=0.025)$. Conclusion. Pretransplant CTC status can guide the administration of pretransplant RFA in HCC patients which can improve PFS in CTC-positive HCC patients.
\end{abstract}

\section{Introduction}

Hepatocellular carcinoma (HCC) is regarded as the most common malignancy and is a leading cause of cancer-related death in the world as the $6^{\text {th }}$ most common worldwide and the $4^{\text {th }}$ leading cancer-related death $[1,2]$. In China, HCC is the fourth most diagnosed cancer and the fourth leading cause of cancer death [3]. Treatment of HCC should be carefully selected to achieve promising outcomes. Hepatic resection (HR) is considered as the first-line treatment for patients without vasculature invasion in China $[4,5]$. However, the numbers of patients who are suitable for radical resection are limited and the overall 5-year recurrence rate remains high [6]. Liver transplantation (LT) has been accepted as the most effective and curative treatment for patients with both HCC and decompensated cirrhosis [7]. In patients for whom transplantation is not an option (tumor size and numbers is beyond Milan criteria), local and systemic treatment are available as bridging therapy for HCC. Thermal ablation, for example, radiofrequency ablation (RFA), is considered as the preferred treatment for local tumor control and used for bridging or downstaging HCC patients before LT [8]. However, the current clinical use of RFA depends on the experiences based on the traditional tumor characteristics, like tumor size, tumor numbers, and alpha-fetoprotein (AFP), and whether 
patients will benefit from pretransplant RFA remains controversial [9-12]. Therefore, it is of great significance to confirm reliable indicators for the guidance of pretransplant RFA for HCC.

Our previous study confirmed that positive circulating tumor cell (CTC) count $(>1 / 3.2 \mathrm{ml}$ whole blood) was related to the early recurrence of patients with HCC after LT and showed that pretransplant CTC status may be useful to predict recurrence [13]. Whether it also is useful to guide the application of pretransplant RFA remains unclear. In this study, we aim to investigate whether CTC status is a clinical indicator for RFA before LT in patients with HCC.

\section{Materials and Methods}

2.1. Patient Enrollment. Between January 2016 and January 2020, 713 patients received LT in our center and 373 patients met inclusion criteria. The inclusion criteria were as follows: 18 to 75 years of age, a diagnosis of HCC confirmed by postoperative pathological examination, and follow-up of more than 1 year. The exclusion criteria were as follows: patients with perioperative or nonrecurrence-related mortality, a diagnosis of other types of tumors, and follow-up of less than 1 year (Figure 1). Afterward, 79 of 373 patients who were tested for CTCs were enrolled in this study. Eighteen patients received only RFA 6 months before LT.

All the procedures were performed in accordance with the ethical standards of the responsible committee on human experimentation (institutional and national) and the Helsinki Declaration of 1964 and later versions. The study was approved by the Institutional Ethics Committee for Clinical Research and Animal Trials of the First Affiliated Hospital of Sun Yat-sen University and an informed consent waiver was granted by the IEC given the retrospective, minimal risk nature of the study. No organs from executed prisoners were transplanted into any of the patients reported in this study.

2.2. Perioperative Management and Follow-Up. The immunosuppressive regimen after LT was tacrolimus $(\mathrm{Tac})+$ mycophenolate mofetil (MMF). The followed-up period was at least 1 year. Postoperative visits were performed on postoperative days (POD) 1-7, POD 14, and each postoperative month (POM). Laboratory tests, imaging examinations, and tumor markers were documented. Routine Doppler ultrasound of the liver graft blood flow and the biliary tract was performed once every 2 days for 7 days. Afterward, imaging studies were performed based on patients' clinical status or laboratory findings. HCC recurrence was diagnosed according to the Guidelines for the Diagnosis and Treatment of Primary Liver Cancer (2019 edition) in China [14]. For deceased patients/patients with recurrences, the date of death/recurrence was used as the last follow-up for overall survival (OS) and progression-free survival (PFS), respectively. The follow-up deadline was January 1, 2021.

2.3. CTCs Detection. The specific method has been described in the previous study [13]. In brief, the samples $(3.2 \mathrm{ml}$ peripheral whole blood collected from a median cubital vein) for CTCs analysis were collected within 1 month before LT. Negative enrichment and imFISH methods were introduced to detect CTCs. The identification of enriched CTCs was performed by imFISH, which combined the FISH probes with chromosome 8 (orange) centromere probes (Abbott Molecular Diagnostics, Des Plaines, IL, USA) and anti-CD45 monoclonal antibodies (Red, Cyttel). To be considered positive, CTCs needed to be hyperdiploid and have the phenotype CEP8+/DAPI+/CD45-. The cutoff value of the CTC count was 1 . It was defined as positive as the CTC count was $\geq 1$.

2.4. RFA Procedures. Indications for pretransplant RFA were primarily evaluated by physicians [15]. To eliminate selection bias, CTC result was not taken into consideration. Briefly, artificial ascites was created firstly by injecting with $100 \mathrm{ml} \mathrm{5 \%} \mathrm{glucose} \mathrm{solution} \mathrm{to} \mathrm{separate} \mathrm{gastrointestinal} \mathrm{tract}$ and liver. An $18 \mathrm{G}$ biopsy needle was used for biopsy of lesion sent for pathological examination. Afterward, the lesions were ablated with anhydrous alcohol and injected with $3 \mathrm{ml}$ anhydrous alcohol by 21G PTC needle. RFA was then performed with Cool-tip ${ }^{\mathrm{TM}}$ electrode needle (ACT2020) for 10-30 minutes. The primary endpoint of RFA is to obtain a complete necrosis of liver tumors and create a safety margin of at least $10 \mathrm{~mm}$ round the external margin of the lesion. Contrast enhanced ultrasound (CEUS) was performed on the second day after ablation to confirm the margin of tumor necrosis.

2.5. Statistical Analysis. All statistical analyses of the data were performed by SPSS version 26.0. All data are expressed as the number and percentage of patients. For comparison between groups, the chi-square and Fisher's exact tests were performed for frequencies and continuous data, respectively. Cox proportional hazards model was performed for multivariate analysis. Overall and disease-free survival were compared using the Kaplan-Meier method. A $P$ value $<0.05$ was considered statistically significant.

\section{Results}

3.1. Baseline Characteristics of Patients with HCC in CTC-Test Group. To eliminate selection bias, we compared baseline data between the CTC-test group and no CTC-test group (Supplementary Table 1) and there were no significant differences in age, gender, AFP, diagnosis with cirrhosis, and TNM staging between groups $(P>0.05)$. Baseline characteristics of 79 patients enrolled in this study are presented in Table 1. The median follow-up time was 15.7 and 17.3 months for PFS and OS, respectively. Of the 79 patients, 42 patients (53.2\%) were detected as CTC-positive (>1/3.2 ml whole blood) and 18 (22.8\%) patients received pretransplant RFA only. Besides that, 28 (35.4\%) patients did not receive any pre-LT treatment, $3(3.7 \%)$ patients received hepatic resection only, $20(25.3 \%)$ patients received transcatheter arterial chemoembolization (TACE) only, and 10 patients (12.6\%) received combined pre-LT treatments. Fifteen 


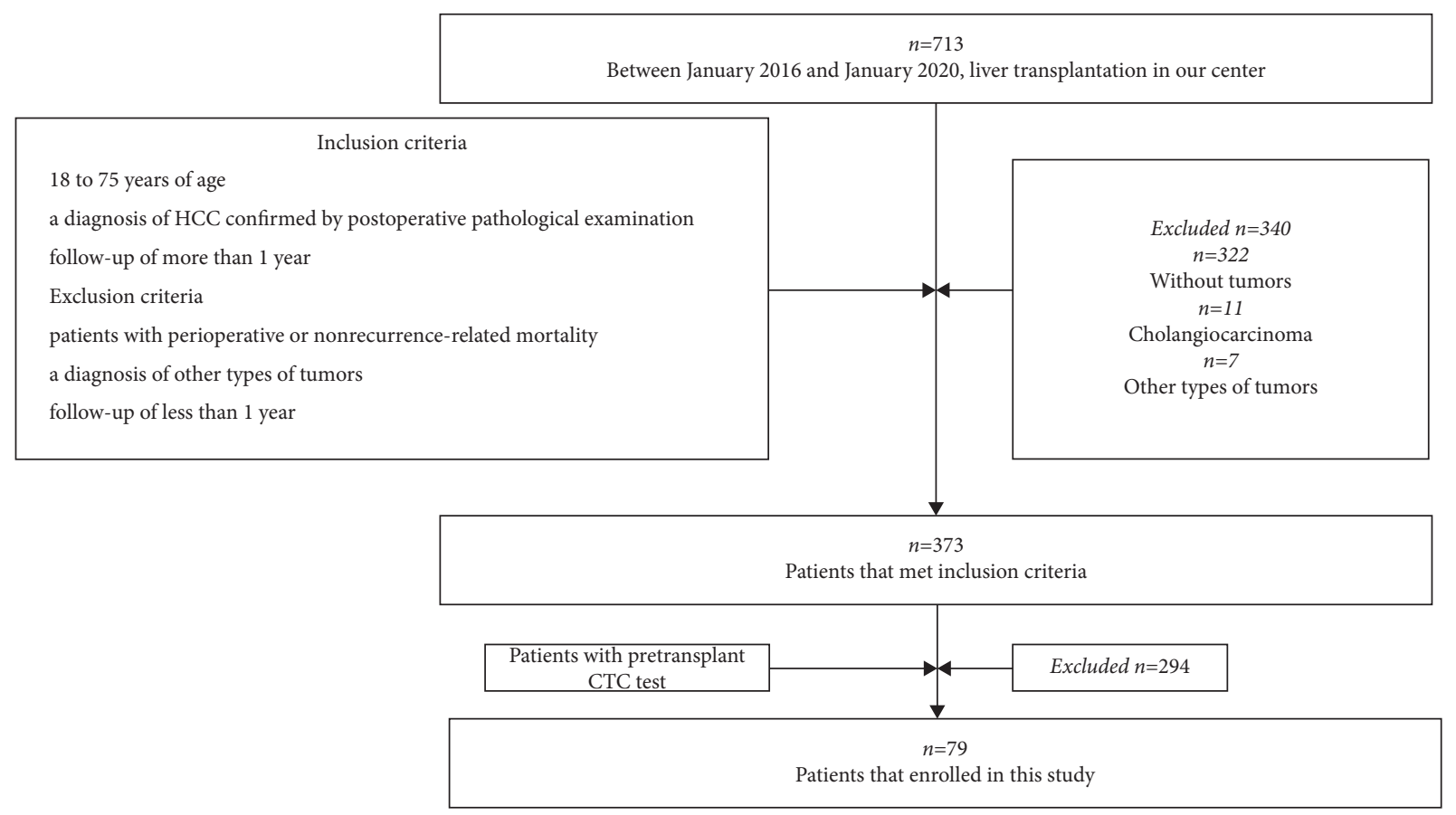

FIGURE 1: Flowchart for patients' selection in this study.

patients $(19.0 \%)$ had multinodular tumors and 31 (39.2\%) patients had tumors of larger size $(>3 \mathrm{~cm})$. Most patients were diagnosed with liver cirrhosis $(92.4 \%)$ and hepatic B virus (HBV) infection (82.3\%).

3.2. CTC Result Is Related to the Early Recurrence of Patients with HCC after LT. Analysis of the 79 patients revealed that $20(25.3 \%)$ patients had a recurrence after LT (Table 2). Fifteen of $20(75 \%)$ patients with recurrence and 27 of 59 (45.7\%) patients without recurrence were positive for CTCs, respectively. The results showed that recurrence was correlated with CTC count $\left(\chi^{2}=5.128 P=0.024\right)$, tumor number $\left(\chi^{2}=4.464, P=0.035\right)$, liver cirrhosis $\left(\chi^{2}=11.559\right.$, $P=0.001)$, Milan criteria $\left(\chi^{2}=8.773, P=0.003\right)$, and University of California San Francisco (UCSF) criteria $\left(\chi^{2}=10.225, P=0.001\right)$, while there were no significant differences in other groups like preoperative AFP $\left(\chi^{2}=1.328, P=0.249\right)$. The Kaplan-Meier analysis revealed that CTC-positive patients had a lower PFS rate compared with CTC-negative patients $(P=0.0257$; Figure 2(a)). However, the OS rate seemed to be similar and not significantly different between CTC-negative and CTC-positive groups $(P=0.5543$, Figure $2(\mathrm{~b}))$.

3.3. Pretransplant RFA Improves PFS in CTC-Positive Patients. Baseline characteristics in HCC patients with or without RFA are shown in Table 3 and no significant differences were found between groups. The association between pretransplant RFA and posttransplant tumor recurrence was analyzed in HCC patients stratified be CTC status. During the follow-up period, recurrence was observed in 15 of 42 CTC-positive patients and 5 of 37 CTC- negative patients, respectively. For CTC-positive patients, the PFS rate of pretransplant RFA group were significantly higher than non-RFA group ( $100 \%$ vs. $46.7 \%, P=0.0236$; Figure $2(\mathrm{c}))$, whereas the OS rates between the groups were similar (87.5\% vs. $83.3 \%, P=0.5543$; Figure $2(d))$. For CTCnegative patients, both PFS rate and OS rate were similar and without significant differences $(P=0.6636$ and 0.0677 , respectively; Figures 2(e) and 2(f)). We also had the comparison between RFA group and nontreatment group in CTC-positive patients. The PFS rate of pretransplant RFA group was significantly higher than nontreatment group (nontreatment means LT directly after diagnosis without other preoperative treatment, $100 \%$ vs. $46.7 \%, P=0.0346$; Figure $2(\mathrm{~g}))$, and the OS rates between the groups were similar $(87.5 \%$ vs. $80 \%, P=0.6277$; Figure $2(\mathrm{~h}))$.

Furthermore, the predictive value of CTC-positive for benefit of pretransplant RFA was evaluated within clinical subgroups (Figure 3). The result showed that the PFS rates were higher in patients with pretransplant RFA. However, no significant differences were found between patients with/ without pretransplant RFA in these subgroups. The Kaplan-Meier survival analyses for clinical subgroups are shown in Figures 3(a)-3(h). The efficacy of RFA to PFS and OS in CTC-positive HCC patients were also evaluated in multivariate analysis. The result showed that pretransplant RFA was the independent factor for PFS but not for OS $(P=0.025$ and 0.382 , respectively; Table 4$)$.

\section{Discussion}

LT has been regarded as the only curative method for patients with HCC. However, posttransplant tumor recurrence was the major limitation for the survival of these patients $[16,17]$. RFA is widely used for bridging or downstaging 
TABLE 1: Baseline characteristics of HCC patients for the entire study.

\begin{tabular}{|c|c|c|c|}
\hline \multirow{2}{*}{ Variable } & & \multicolumn{2}{|c|}{$N=79$} \\
\hline & & $n$ & $\%$ \\
\hline \multirow{2}{*}{ Gender } & Male & 74 & 93.7 \\
\hline & Female & 5 & 6.3 \\
\hline \multirow{2}{*}{ Age (years) } & $>50$ & 47 & 59.5 \\
\hline & $\leq 50$ & 32 & 40.5 \\
\hline \multirow{2}{*}{ CTC count } & $>1$ & 42 & 53.2 \\
\hline & $\leq 1$ & 37 & 46.8 \\
\hline \multirow{2}{*}{ Tumor number } & $>3$ & 15 & 19.0 \\
\hline & $\leq 3$ & 64 & 81.0 \\
\hline \multirow{2}{*}{ Tumor diameter $(\mathrm{cm})$} & $>3$ & 31 & 39.2 \\
\hline & $\leq 3$ & 48 & 60.8 \\
\hline \multirow{2}{*}{ PVT } & Yes & 16 & 20.3 \\
\hline & No & 63 & 79.7 \\
\hline \multirow{2}{*}{ MVI } & Yes & 15 & 19.0 \\
\hline & No & 64 & 81.0 \\
\hline \multirow{2}{*}{ Edmonson stage } & I-II & 48 & 60.8 \\
\hline & III-IV & 31 & 39.2 \\
\hline \multirow{2}{*}{ Liver cirrhosis } & Yes & 73 & 92.4 \\
\hline & No & 6 & 7.6 \\
\hline \multirow{2}{*}{ Milan criteria } & Yes & 46 & 58.2 \\
\hline & No & 33 & 41.7 \\
\hline \multirow{2}{*}{ UCSF criteria } & Yes & 51 & 64.6 \\
\hline & No & 28 & 35.4 \\
\hline \multirow{2}{*}{ HBsAg $(+)$} & Yes & 65 & 82.3 \\
\hline & No & 14 & 17.7 \\
\hline \multirow{2}{*}{$\operatorname{AFP}(\mathrm{ng} / \mathrm{ml})$} & $>400$ & 20 & 25.3 \\
\hline & $\leq 400$ & 59 & 74.7 \\
\hline \multirow{3}{*}{ TNM stage } & I & 10 & 12.7 \\
\hline & II & 23 & 29.1 \\
\hline & III-IV & 46 & 58.2 \\
\hline \multirow{2}{*}{ Pretransplantation treatment } & Yes & 51 & 64.6 \\
\hline & No & 28 & 35.4 \\
\hline \multirow{2}{*}{ RFA only } & Yes & 18 & 22.8 \\
\hline & No & 61 & 77.2 \\
\hline \multirow{2}{*}{ Recurrence } & Yes & 20 & 25.3 \\
\hline & No & 59 & 74.7 \\
\hline
\end{tabular}

AFP: alpha-fetoprotein; CTC: circulating tumor cells; HBsAg: hepatitis B surface antigen; HCC: hepatocellular carcinoma; MVI: microvascular invasions; PVT: portal vein thrombosis; RFA: radiofrequency ablation; UCSF: University of California San Francisco.

HCC patients before LT [18]. Whether patients will benefit from pretransplant RFA for the lack of reliable indicators remains controversial [19-21]. In this retrospective study, we aimed to investigate whether the CTC result could indicate the application of pretransplant RFA for HCC patients. Overall, our result showed that pretransplant RFA reduces recurrence effectively in CTC-positive patients with HCC. However, For CTC-negative patients, pretransplant RFA cannot reduce both PFS and OS rates. Therefore, the pretransplant CTC result may be used as an indicator for RFA before LT for patients with HCC.

Recurrences are the most negative factor affecting survival for LT patients with HCC $[7,22]$. The main cause of recurrence is tumor cell dissemination via blood vessel infiltration [23]. In our study, recurrence after LT was related to CTC count, Milan criteria, and UCSF criteria. The recurrence of LT within the Milan criteria was $13.0 \%$, and it is better than $43.6 \%$ for those beyond the Milan criteria. The recurrence of LT within the UCSF criteria was $13.7 \%$ and it is also better than $46.4 \%$ for those beyond UCSF criteria. This indicates that Milan criteria and UCSF criteria are promising criteria for favorable outcomes [24-26]. However, in China, patients tend to have HR or conservative treatment due to economic or ideological reasons, even if tumors are detected early. LT would be considered only when other treatments were ineffective or if the tumor progressed. Therefore, finding a method to predict the prognosis after LT is of great significance. Imaging, pathological examinations, and common serum markers like AFP have their limitations in diagnostic accuracy and sensitivity and a novel diagnostic method is needed [27]. CTCs were first discovered and described by Ashworth et al. in 1869 [28]. Vona et al. first explored the prognostic value of blood CTCs in patients with HCC and it was related to prognosis and recurrence in patients with HCC [29]. CTC detection can be applied as a method for early cancer detection and prediction of recurrence or metastasis risk [30-32]. Compared with the conventional clinicopathological index like AFP, CTC has the advantage of predicting microvascular invasion and dynamical detection [33]. Castro-Giner and Aceto showed in their study that it can serve as a promising tool to provide insights into the biology of metastatic cancers and with potential for use in liquid biopsy-based personalized cancer treatment [34]. Ramirez et al. showed in their study that CTC was an essential key for the management of the patients with HCC on the waiting list for liver transplantation [35]. Zhou et al. found CTCs can indicate the prognosis of HCC for its efficacy in predicting microvascular invasion [36]. Our previous study also revealed that CTC-positive patients had a worse prognosis after LT than CTC-negative patients [13]. In the current study, it remains unclear whether patients can benefit from RFA by achieving a high degree of tumor necrosis before LT [37]. Agopian et al. showed in their study including 3601 recipients of LT that none of the significant differences were identified in survival between patients with/without locoregional treatment (LRT) prior to LT [8]. However, in his study, only $10 \%$ of patients received ablation therapy and only $10 \%$ of patients had HCC secondary to HBV. Different types of HCC have different responses to the preoperative treatment. A small percentage of pretransplant RFA and HBV related HCC might be the reason for its failure to get comparable results. Our results showed that pretransplant RFA improves PFS effectively in CTC-positive patients with HCC. However, in clinical subgroups, significant differences were not found between patients with/without pretransplant RFA. We consider these results in traditional clinical subgroups were not in conflict with previous studies $[38,39]$, for CTC result is a novel biomarker to evaluate the risk of recurrence and may be a complementary biomarker of traditional clinical indicators to pretransplant RFA in HCC patients. It can be applied to the guidance for the downstaging treatment before LT [40]. In addition, the CTC result can be used to guide the 
TABLE 2: Analysis of relevant factors for recurrence of HCC in 79 patients.

\begin{tabular}{|c|c|c|c|c|c|}
\hline \multirow{2}{*}{ Variable } & & \multicolumn{4}{|c|}{$N=79$} \\
\hline & & Recurrence $(n=20)$ & Nonrecurrence $(n=59)$ & $x^{2}$ & $P$ value \\
\hline \multirow{2}{*}{ Gender, $n(\%)$} & Male & $19(24.1)$ & $55(69.6)$ & \multirow{2}{*}{0.08} & \multirow{2}{*}{0.778} \\
\hline & Female & $1(1.3)$ & $4(5.1)$ & & \\
\hline \multirow{2}{*}{ Age (years), $n(\%)$} & $>50$ & $11(13.9)$ & $36(45.6)$ & \multirow{2}{*}{0.224} & \multirow{2}{*}{0.636} \\
\hline & $\leq 50$ & $9(11.4)$ & $23(29.1)$ & & \\
\hline \multirow{2}{*}{ CTC count, $n(\%)$} & $>1$ & $15(19.0)$ & $27(30.4)$ & \multirow{2}{*}{5.128} & \multirow{2}{*}{0.024} \\
\hline & $\leq 1$ & $5(6.3)$ & $32(40.5)$ & & \\
\hline \multirow{2}{*}{ Tumor number, $n(\%)$} & $>3$ & $7(8.9)$ & $8(10.1)$ & \multirow[b]{2}{*}{4.464} & \multirow{2}{*}{0.035} \\
\hline & $\leq 3$ & $13(16.5)$ & $51(64.6)$ & & \\
\hline \multirow{2}{*}{ Tumor diameter $(\mathrm{cm}), n(\%)$} & $>3$ & $10(12.7)$ & $19(24.1)$ & \multirow{2}{*}{2.036} & \multirow{2}{*}{0.154} \\
\hline & $\leq 3$ & $10(12.7)$ & $40(50.6)$ & & \\
\hline \multirow{2}{*}{ PVT, $n(\%)$} & Yes & $6(7.6)$ & $10(12.7)$ & \multirow{2}{*}{1.575} & \multirow{2}{*}{0.209} \\
\hline & No & $14(17.7)$ & $49(62.0)$ & & \\
\hline \multirow{2}{*}{ MVI, $n(\%)$} & Yes & $7(8.9)$ & $10(12.7)$ & \multirow{2}{*}{2.882} & \multirow{2}{*}{0.090} \\
\hline & No & $13(16.5)$ & $49(62.0)$ & & \\
\hline \multirow{2}{*}{ Edmonson stage, $n(\%)$} & I-II & $9(11.4)$ & $39(49.4)$ & \multirow{2}{*}{2.790} & \multirow{2}{*}{0.095} \\
\hline & III-IV & $11(13.9)$ & $20(25.3)$ & & \\
\hline \multirow{2}{*}{ Liver cirrhosis, $n$ (\%) } & Yes & $15(19.0)$ & $58(73.4)$ & \multirow{2}{*}{11.559} & \multirow{2}{*}{0.001} \\
\hline & No & $5(6.3)$ & $1(1.3)$ & & \\
\hline \multirow{2}{*}{ Milan criteria, $n(\%)$} & Yes & $6(7.6)$ & $40(50.6)$ & & \\
\hline & No & $14(17.7)$ & $19(24.0)$ & $8.1 / 3$ & 0.003 \\
\hline & Yes & $7(8.8)$ & $44(55.6)$ & & 0.001 \\
\hline UCSF criteria, $n(\%)$ & No & $13(16.4)$ & $15(18.9)$ & 10.225 & 0.001 \\
\hline $\mathrm{HBsAg}(+), n(\%)$ & Yes & $16(20.3)$ & $49(62.0)$ & & 0.757 \\
\hline HBSAg (+), $n$ (\%) & No & $4(5.1)$ & $10(12.7)$ & 0.095 & 0.757 \\
\hline RFA only, $n(\%)$ & Yes & $2(2.5)$ & $16(20.3)$ & 2.448 & 0.115 \\
\hline КГА Оाту, $n$ (\%) & No & $18(22.8)$ & $43(54.4)$ & 2.440 & 0.115 \\
\hline AFP (ng/ml), n (\%) & $>400$ & $7(8.9)$ & $13(16.5)$ & 1328 & 0.249 \\
\hline AFP (ng/mI), $n$ (\%) & $\leq 400$ & $13(16.5)$ & $46(58.2)$ & 1.328 & 0.249 \\
\hline & I & 0 & $10(12.7)$ & & \\
\hline TNM stage, $n(\%)$ & II & $4(5.1)$ & $19(24.1)$ & 6.334 & 0.042 \\
\hline & III-IV & $16(20.3)$ & $30(38.0)$ & & \\
\hline
\end{tabular}

Bold $P$ values indicate statistical significance. AFP: alpha-fetoprotein; CTC: circulating tumor cells; HBsAg: hepatitis B surface antigen; HCC: hepatocellular carcinoma; MVI: microvascular invasions; PVT: portal vein thrombosis; RFA: radiofrequency ablation; UCSF: University of California San Francisco.

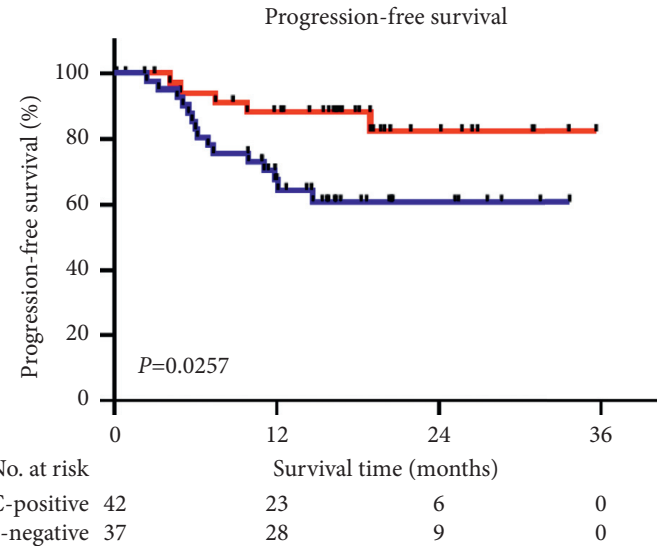

$\longrightarrow$ CTC-positive
$\longrightarrow$ CTC-negative

(a)

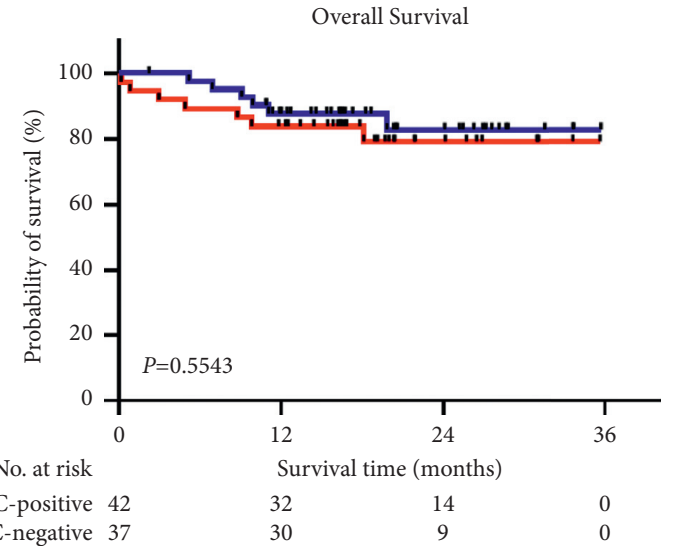

$\longrightarrow$ CTC-positive

(b)

Figure 2: Continued. 


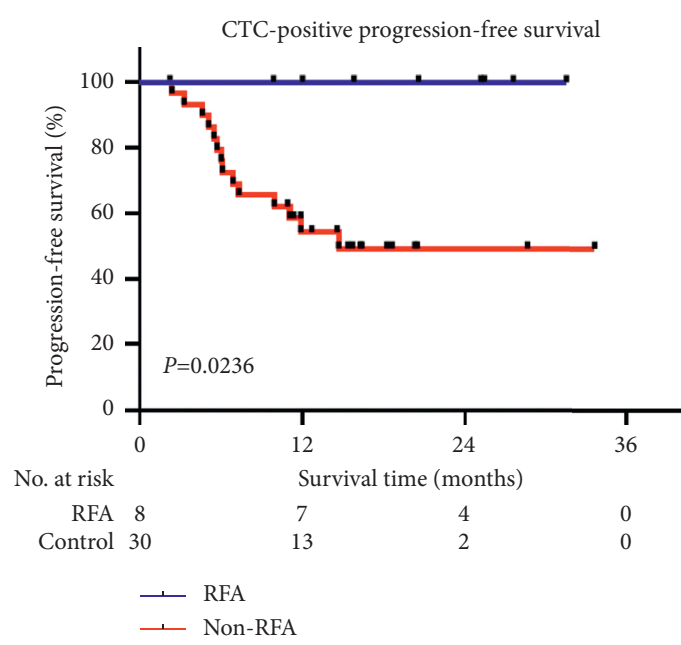

(c)

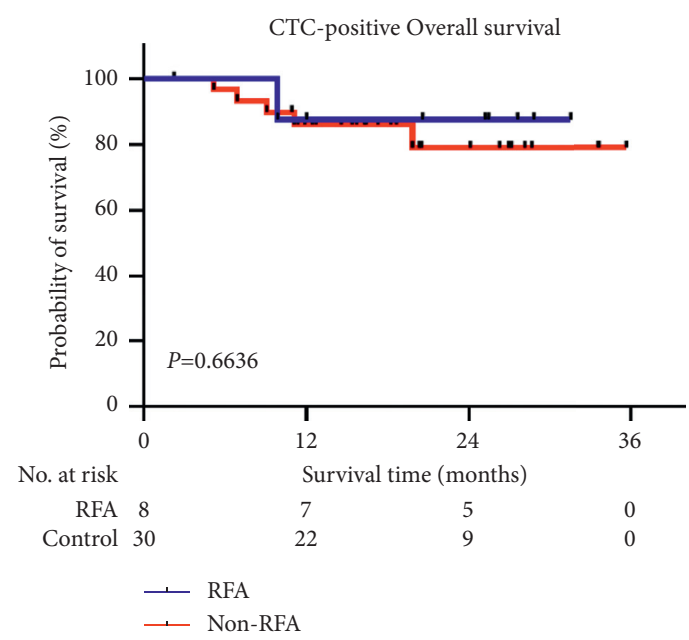

(e)

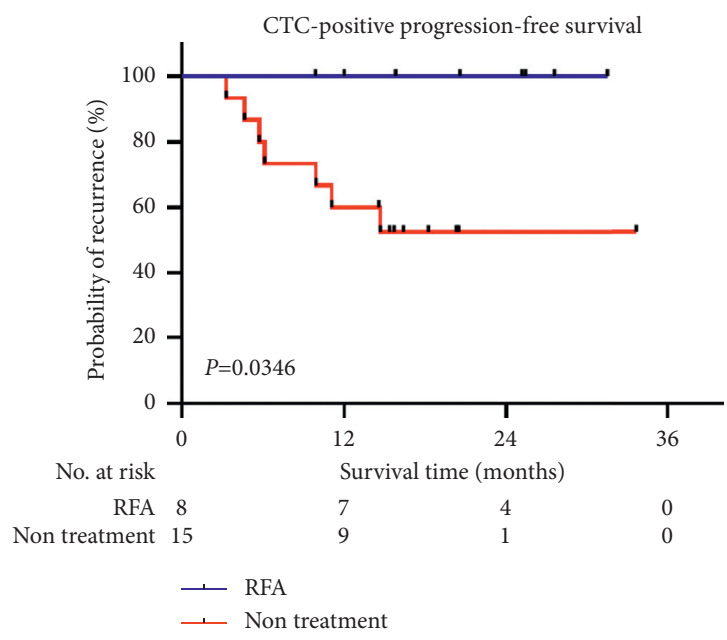

(g)

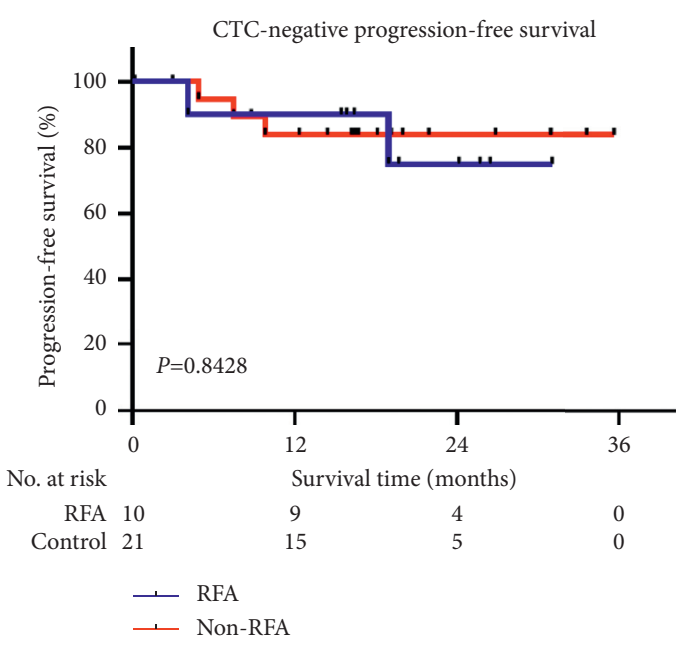

(d)

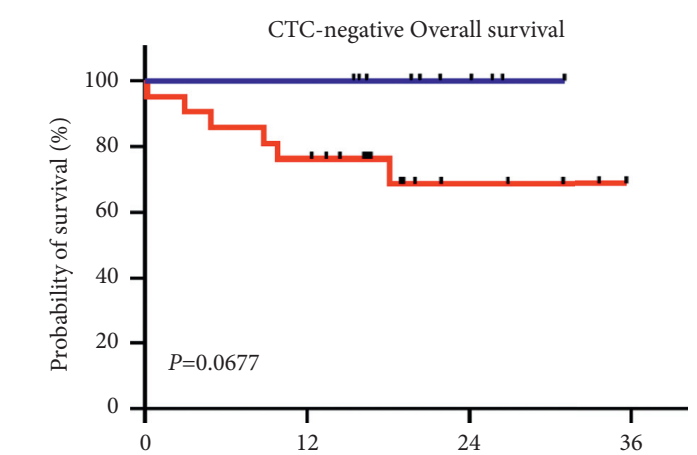

No. at risk

Survival time (months)

$\begin{array}{rrrrr}\text { RFA } & 10 & 10 & 4 & 0 \\ \text { Control } & 21 & 16 & 5 & 0\end{array}$

- RFA

- Non-RFA

(f)

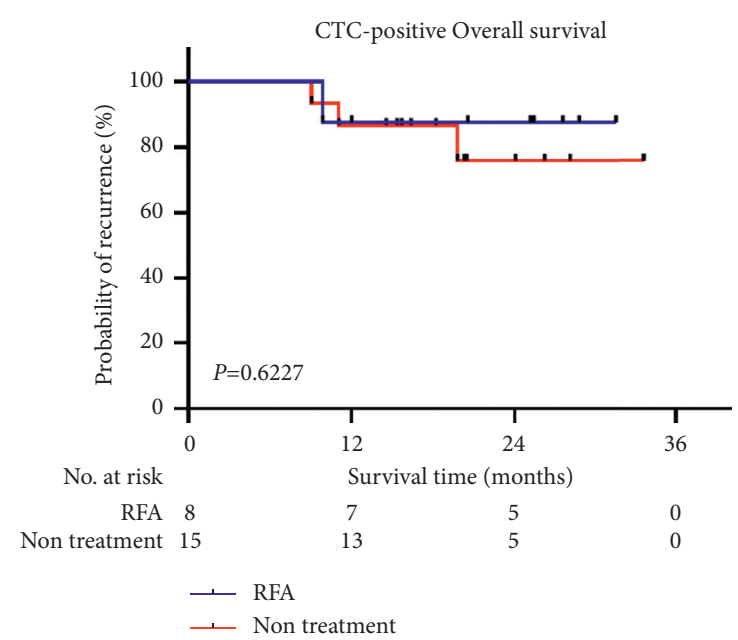

(h)

FIGURE 2: Comparison of PFS and OS between different groups of HCC patients. (a) PFS between CTC-positive and CTC-negative groups; (b) OS between CTC-positive and CTC-negative groups; (c) PFS between RFA and non-RFA groups in CTC-positive HCC patients; (d) PFS between RFA and non-RFA groups in CTC- negative HCC patients; (e) OS between RFA and non-RFA groups in CTC-positive HCC patients; (f) OS between RFA and non-RFA groups in CTC-negative HCC patients. (g) PFS between RFA and nontreatment groups in CTCpositive HCC patients; (h) OS between RFA and nontreatment groups in CTC-positive HCC patients. 
TABLE 3: Baseline characteristics in HCC patients with/without RFA.

\begin{tabular}{|c|c|c|c|c|c|}
\hline \multirow{2}{*}{ Variable } & & \multicolumn{4}{|c|}{$N=79$} \\
\hline & & RFA $(n=18)$ & Non-RFA $(n=61)$ & $\chi^{2}$ & $P$ value \\
\hline \multirow{2}{*}{ Gender, $n(\%)$} & Male & $16(20.3)$ & $58(73.4)$ & \multirow{2}{*}{0.899} & \multirow{2}{*}{0.343} \\
\hline & Female & $2(2.5)$ & $3(3.8)$ & & \\
\hline \multirow{2}{*}{ Age (years), $n(\%)$} & $>50$ & $8(10.1)$ & $39(49.4)$ & \multirow{2}{*}{2.191} & \multirow{2}{*}{0.139} \\
\hline & $\leq 50$ & $10(12.7)$ & $22(27.8)$ & & \\
\hline \multirow{2}{*}{ CTC count, $n(\%)$} & $>1$ & $8(10.1)$ & $34(43.0)$ & \multirow{2}{*}{0.712} & \multirow{2}{*}{0.399} \\
\hline & $\leq 1$ & $10(12.7)$ & $27(34.2)$ & & \\
\hline \multirow{2}{*}{ Tumor number, $n(\%)$} & $>3$ & $1(1.3)$ & $14(17.7)$ & \multirow{2}{*}{2.734} & \multirow{2}{*}{0.098} \\
\hline & $\leq 3$ & $17(21.5)$ & $47(59.5)$ & & \\
\hline \multirow{2}{*}{ Tumor diameter $(\mathrm{cm}), n(\%)$} & $>3$ & $6(7.6)$ & $9(11.4)$ & \multirow{2}{*}{3.119} & \multirow{2}{*}{0.077} \\
\hline & $\leq 3$ & $12(15.2)$ & $52(65.8)$ & & \\
\hline \multirow{2}{*}{$\mathrm{PVT}, n(\%)$} & Yes & $3(3.8)$ & $13(16.5)$ & \multirow{2}{*}{0.186} & \multirow{2}{*}{0.667} \\
\hline & No & $15(19.0)$ & $48(60.8)$ & & \\
\hline \multirow{2}{*}{ MVI, $n(\%)$} & Yes & $3(3.8)$ & $12(15.2)$ & \multirow{2}{*}{0.082} & \multirow{2}{*}{0.775} \\
\hline & No & $15(19.0)$ & $49(62.0)$ & & \\
\hline \multirow{2}{*}{ Edmonson stage, $n(\%)$} & I-II & $11(13.9)$ & $37(46.8)$ & \multirow{2}{*}{0.001} & \multirow{2}{*}{0.972} \\
\hline & III-IV & $7(8.9)$ & $24(30.4)$ & & \\
\hline \multirow{2}{*}{ Liver cirrhosis, $n(\%)$} & Yes & $17(21.5)$ & $56(71.0)$ & \multirow{2}{*}{0.138} & \multirow{2}{*}{0.710} \\
\hline & No & $1(1.3)$ & $5(6.3)$ & & \\
\hline \multirow{2}{*}{ Milan criteria, $n(\%)$} & Yes & $14(11.4)$ & $32(40.5)$ & \multirow{2}{*}{3.663} & \\
\hline & No & $4(5.0)$ & $29(36.7)$ & & 0.056 \\
\hline UCSF criteria $n$ (\%) & Yes & $15(18.9)$ & $36(45.5)$ & 3592 & 0058 \\
\hline UCSF criteria, $n(\%)$ & No & $3(3.7)$ & $25(31.6)$ & 3.592 & 0.058 \\
\hline $\mathrm{HB}_{\mathrm{A}} \mathrm{Ag}(+) n(0)$ & Yes & $14(17.7)$ & $51(64.6)$ & 0324 & 0,560 \\
\hline HBsAg (+), $n$ (\%) & No & $4(5.1)$ & $10(12.7)$ & 0.324 & 0.569 \\
\hline $\mathrm{AFP}(\mathrm{ng} / \mathrm{ml}), n(\%)$ & $>400$ & $3(3.8)$ & $17(21.5)$ & 0.922 & 0337 \\
\hline AFP (ng/ml), $n(\%)$ & $\leq 400$ & $15(19.0)$ & $44(55.7)$ & 0.922 & 0.337 \\
\hline & I & $1(1.3)$ & $9(11.4)$ & & \\
\hline TNM stage, $n(\%)$ & II & $7(8.9)$ & $16(20.3)$ & 1.723 & 0.423 \\
\hline & III-IV & $10(12.7)$ & $36(45.6)$ & & \\
\hline
\end{tabular}

AFP: alpha-fetoprotein; CTC: circulating tumor cells; HBsAg: hepatitis B surface antigen; HCC: hepatocellular carcinoma; MVI: microvascular invasions; PVT: portal vein thrombosis; RFA: radiofrequency ablation; UCSF: University of California San Francisco.

pretransplant management for HCC patients. In our study, CTCs were detected in 42 of 79 (53.2\%) patients before LT. The sensitivity and specificity of CTCs detection were $75 \%$ and $54.2 \%$, respectively. The results showed that the CTCs test had good sensitivity and specificity so it could be helpful to predict recurrence. An additional pretransplant RFA may not be necessarily needed for CTC-negative patients. A further prospective, multicenter, and large population study is needed to investigate the value of the CTC result as an indicator in guiding pretransplant treatment.

RFA was first applied and described by Rossi et al. in 1993 [41]. In China, the use of RFA for HCC was quickly developed in recent years. Compared with HR, RFA is minimally invasive and has lower morbidity and mortality rates, especially in cases with impaired liver functions. However, the tumor size and stage are important factors for the outcome of RFA [42, 43]. Yan et al. showed in their study that a larger tumor size $(>5 \mathrm{~cm})$ would result in a less complete necrosis rate and RFA alone for HCC is limited [44]. The combination of RFA and other methods would have further benefits for patients. RFA can be used for bridging or downstaging HCC patients before LT; therefore, it may help to prolong time on the waiting list and reduce the waiting list mortality rate [45]. The current clinical use of RFA depends on the experiences based on the traditional tumor characteristics and a novel biomarker to guide pretransplant treatment is of great significance. Our result showed that transplant RFA improves PFS effectively of patients in the status of CTCpositive. For CTC-negative patients, pretransplant RFA did not improve the early PFS rate, and this suggested that RFA may not be necessary for such patients. For the overall survival comparison, it seems like the CTC-negative patients with pretransplant RFA got a better OS than non-RFA counterparts ( $100 \%$ vs. $76.2 \%)$, however without significant difference $(P=0.0677)$. Small sample size and short follow-up period may be the reason. Enlarging sample scale and prolonging follow-up period are needed for convincing result. Furthermore, we made a multivariate analysis and figured out that pretransplant RFA was the independent factor for PFS.

Our study has limitations. First, the sample size is small and from a single-center institution. Larger multicenter studies are needed to determine whether pretransplant RFA 


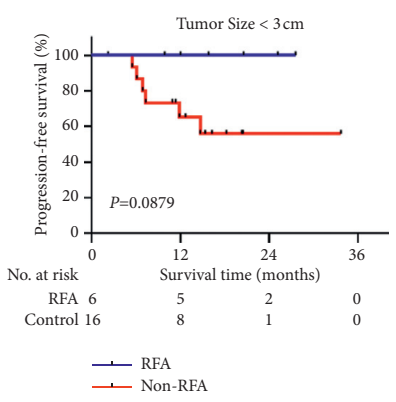

(a)

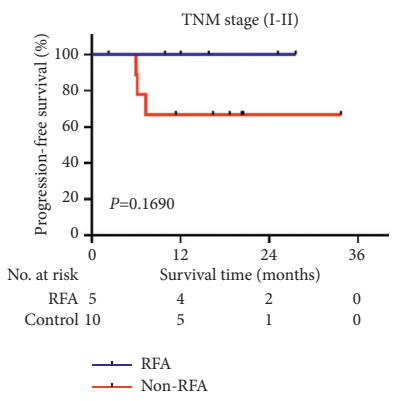

(e)

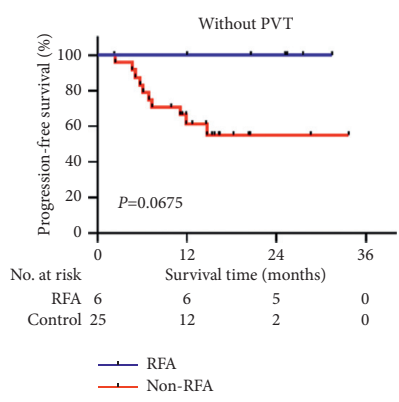

(b)

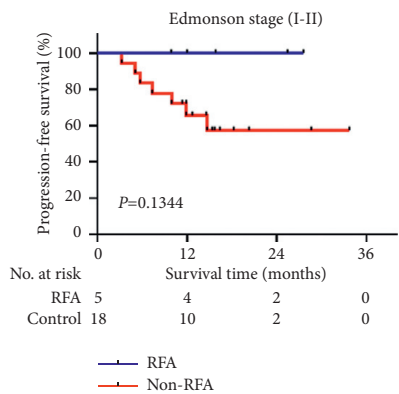

(f)

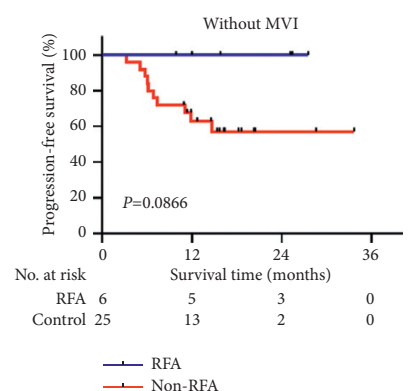

(c)

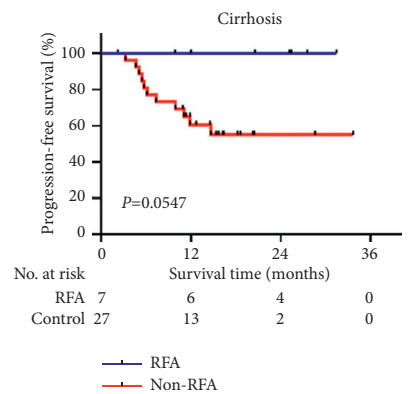

(g)

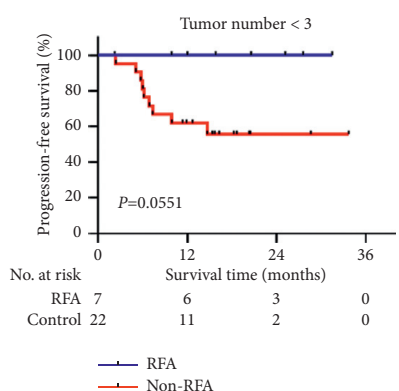

(d)

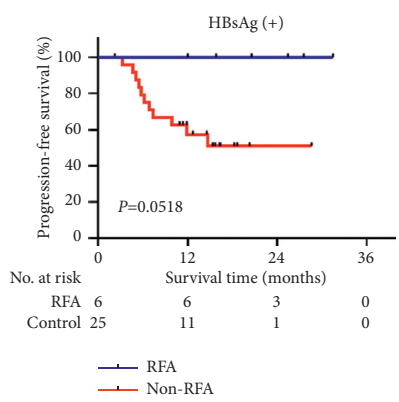

(h)

FIgURe 3: Kaplan-Meier analysis of PFS in subgroups of CTC-positive HCC patients. (a) Tumor size $<3 \mathrm{~cm}$. (b) Without PVT. (c) Without MVI. (d) Tumor number <3. (e) Tumor stage (I-II). (f) Edmonson stage (I-II). (g) With cirrhosis. (h) HBsAg (+). PVT: portal vein thrombosis; MVI: microvascular invasions; HBsAg: hepatitis B surface antigen.

TABLE 4: Multivariate analysis to identify independent risk factors of progression-free survival and overall survival in CTC-positive HCC patients.

\begin{tabular}{|c|c|c|c|c|}
\hline \multirow{2}{*}{ Variable } & \multicolumn{2}{|c|}{ Progression-free survival } & \multicolumn{2}{|c|}{ Overall survival } \\
\hline & HR $(95 \% \mathrm{CI})$ & $P$ value & HR $(95 \% \mathrm{CI})$ & $P$ value \\
\hline Tumor number $(>3)$ & $3.059(0.672 \sim 13.394)$ & 0.148 & $0.380(0.005 \sim 30.134)$ & 0.664 \\
\hline Tumor size $(>3 \mathrm{~cm})$ & $0.348(0.051 \sim 2.395)$ & 0.284 & $2098.606\left(0 \sim 5.316^{*} 10^{9}\right)$ & 0.934 \\
\hline Edmonson stage (III-IV) & $0.693(0.136 \sim 3.523)$ & 0.659 & $1.297(0.068 \sim 24.657)$ & 0.863 \\
\hline TNM stage (III-IV) & $1.394(0.340 \sim 5.721)$ & 0.644 & $8.755(0.272 \sim 281.503)$ & 0.220 \\
\hline $\operatorname{AFP}(>400 \mathrm{ng} / \mathrm{ml})$ & $2.047(0.340 \sim 12.329)$ & 0.434 & $1.944(0.04 \sim 91.723)$ & 0.735 \\
\hline $\operatorname{HBsAg}(+)$ & $0.348(0.054 \sim 2.259)$ & 0.269 & $0.045(0.000 \sim 61.85)$ & 0.216 \\
\hline Pretransplant RFA (yes) & $0.076(0.008 \sim 0.724)$ & 0.025 & $0.140(0.002 \sim 11.449)$ & 0.382 \\
\hline
\end{tabular}

Bold $P$ values indicate statistical significance. AFP: alpha-fetoprotein; CTC: circulating tumor cells; HBsAg: hepatitis B surface antigen; HCC: hepatocellular carcinoma; MVI: microvascular invasions; PVT: portal vein thrombosis; RFA: radiofrequency ablation; UCSF: University of California San Francisco.

can improve PFS in patients with HCC. Second, the value of postoperative CTCs in guiding pretransplant RFA should be analyzed in further study. For future studies, the 3-year and 5 -year PFS and OS values should be calculated to obtain more convincing conclusions.

\section{Conclusion}

In conclusion, this study provides evidence that CTC results can be used for guiding pretransplant RFA for patients with HCC. Therefore, the CTC result is a potentially promising biomarker and clinical indicator for the administration of pretransplant RFA for HCC patients.

\section{Abbreviations}

AFP: Alpha-fetoprotein
CTC: Circulating tumor cell

HCC: Hepatocellular carcinoma

HR: Hepatic resection

LRT: Locoregional treatment

MMF: Mycophenolate mofetil

OS: Overall survival

PFS: Progression-free survival

POD: Postoperative day

RFA: Radiofrequency ablation

Tac: Tacrolimus

UCSF: University of California San Francisco.

\section{Data Availability}

The data that support the findings of this study are available from the corresponding author upon reasonable request. 


\section{Conflicts of Interest}

No conflicts of interest exist in the submission of this manuscript.

\section{Authors' Contributions}

Zhitao Chen and Tielong Wang contributed equally to this work. Xiaoshun He and Weiqiang Ju contributed to conception and design. Maogen Chen and Weiqiang Ju provided administrative support. Zhitao Chen and Xitao Hong provided study materials or patients. Zhitao Chen and Tielong Wang were involved in collection and assembly of data. Tielong Wang and Jia Yu analyzed and interpreted data. Zhitao Chen, Tielong Wang, Chuanbao Chen, Xitao Hong, Yihao Ma, Yiwen Guo, Changjun Huang, Xiaoshun $\mathrm{He}$, Weiqiang Ju, and Maogen Chen contributed to manuscript writing. Zhitao Chen, Tielong Wang, Xitao Hong, Jia Yu, Yihao Ma, Yiwen Guo, Changjun Huang, Xiaoshun He, Weiqiang Ju, and Maogen Chen approved the final manuscript.

\section{Acknowledgments}

This work was supported by the National Natural Science Foundation of China (81401324 and 81770410), the Science and Technology Planning Project of Guangdong Province (2016A020215048), the Guangdong Provincial Key Laboratory of Organ Donation and Transplant Immunology (2013A061401007), the Guangdong Basic and Applied Basic Research Foundation (2020A1515011557 and 2020A1515010903), and the Guangdong Provincial International Cooperation Base of Science and Technology (Organ Transplantation) (2015B050501002), China.

\section{Supplementary Materials}

Table 1: baseline data comparison between no CTC-test and CTC-test patients with HCC. (Supplementary Materials)

\section{References}

[1] A. Forner, M. Reig, and J. Bruix, "Hepatocellular carcinoma," The Lancet, vol. 391, no. 10127, pp. 1301-1314, 2018.

[2] M. Gryziak, K. Woźniak, L. Kraj, and R. Stec, "Milestones in the treatment of hepatocellular carcinoma: a systematic review," Critical Reviews in Oncology, vol. 157, Article ID 103179, 2021.

[3] W. Chen, R. Zheng, P. D. Baade et al., "Cancer statistics in China, 2015," CA: A Cancer Journal for Clinicians, vol. 66, no. 2, pp. 115-132, 2016.

[4] J. M. Llovet, C. Brú, and J. Bruix, "Prognosis of hepatocellular carcinoma: the BCLC staging classification," Seminars in Liver Disease, vol. 19, no. 3, pp. 329-338, 1999.

[5] T. Ishizawa, K. Hasegawa, T. Aoki et al., "Neither multiple tumors nor portal hypertension are surgical contraindications for hepatocellular carcinoma," Gastroenterology, vol. 134, no. 7, pp. 1908-1916, 2008.

[6] J.-H. Wang, C.-C. Wang, C.-H. Hung, C.-L. Chen, and S.-N. Lu, "Survival comparison between surgical resection and radiofrequency ablation for patients in BCLC very early/early stage hepatocellular carcinoma," Journal of Hepatology, vol. 56, no. 2, pp. 412-418, 2012.

[7] G. Sapisochin and J. Bruix, "Liver transplantation for hepatocellular carcinoma: outcomes and novel surgical approaches," Nature Reviews Gastroenterology \& Hepatology, vol. 14, no. 4, pp. 203-217, 2017.

[8] V. G. Agopian, M. P. Harlander-Locke, R. M. Ruiz et al., "Impact of pretransplant bridging locoregional therapy for patients with hepatocellular carcinoma within milan criteria undergoing liver transplantation," Annals of Surgery, vol. 266, no. 3, pp. 525-535, 2017.

[9] A. B. Benson 3rd, T. A. Abrams, E. Ben-Josef et al., "NCCN clinical practice guidelines in oncology: hepatobiliary cancers," Journal of the National Comprehensive Cancer Network: Journal of the National Comprehensive Cancer Network, vol. 7, no. 4, pp. 350-391, 2009.

[10] Y.-J. Zhang and M. S. Chen, "Role of radiofrequency ablation in the treatment of small hepatocellular carcinoma," World Journal of Hepatology, vol. 2, no. 4, pp. 146-150, 2010.

[11] P. Habibollahi, R. A. Sheth, and E. N. K. Cressman, "Histological correlation for radiofrequency and microwave ablation in the local control of hepatocellular carcinoma (HCC) before liver transplantation: a comprehensive review," Cancers, vol. 13, no. 1, 2020.

[12] F. Özdemir and A. Baskiran, "The importance of AFP in liver transplantation for HCC," Journal of Gastrointestinal Cancer, vol. 51, no. 4, pp. 1127-1132, 2020.

[13] Z. Chen, X. Lin, C. Chen et al., "Analysis of preoperative circulating tumor cells for recurrence in patients with hepatocellular carcinoma after liver transplantation," Annals of Translational Medicine, vol. 8, no. 17, p. 1067, 2020.

[14] G. Qiu, Z. Jin, X. Chen, and J. Huang, "Interpretation of guidelines for the diagnosis and treatment of primary liver cancer (2019 edition) in China," Global Health \& Medicine, vol. 2, no. 5, pp. 306-311, 2020.

[15] F. Zhu and H. Rhim, "Thermal ablation for hepatocellular carcinoma: what's new in 2019," Chinese Clinical Oncology, vol. 8, no. 6, p. 58, 2019.

[16] B. Sarici, B. Isik, and S. Yilmaz, "Management of recurrent HCC after liver transplantation," Journal of Gastrointestinal Cancer, vol. 51, no. 4, pp. 1197-1199, 2020.

[17] E. C. Verna, Y. A. Patel, A. Aggarwal et al., "Liver transplantation for hepatocellular carcinoma: management after the transplant," American Journal of Transplantation, vol. 20, no. 2, pp. 333-347, 2020.

[18] D. Kollmann, N. Selzner, and M. Selzner, "Bridging to liver transplantation in HCC patients," Langenbeck's Archives of Surgery, vol. 402, no. 6, pp. 863-871, 2017.

[19] L. Yu, Z. Dai, Z. Wang, J. Fan, and J. Zhou, "Prognostic indicators for tumor recurrence after liver transplantation in hepatocellular carcinoma and related molecular targeted therapy," Oncology, vol. 81, no. Suppl 1, pp. 116-122, 2011.

[20] Q. Lai, S. Iesari, F. Melandro, G. Mennini, M. Rossi, and J. Lerut, "The growing impact of alpha-fetoprotein in the field of liver transplantation for hepatocellular cancer: time for a revolution," Translational Gastroenterology and Hepatology, vol. 2, no. 9, p. 72, 2017.

[21] T. J. Vogl, L. J. Riegelbauer, E. Oppermann et al., "Early dynamic changes in circulating tumor cells and prognostic relevance following interventional radiological treatments in patients with hepatocellular carcinoma," PLoS One, vol. 16, no. 2, Article ID e0246527, 2021. 
[22] A. Mazzola, A. Costantino, S. Petta et al., "Recurrence of hepatocellular carcinoma after liver transplantation: an update," Future Oncology, vol. 11, no. 21, pp. 2923-2936, 2015.

[23] H. Wang, N. H. Stoecklein, P. P. Lin, and O. Gires, "Circulating and disseminated tumor cells: diagnostic tools and therapeutic targets in motion," Oncotarget, vol. 8, no. 1, pp. 1884-1912, 2017.

[24] N. de'Angelis, F. Landi, M. C. Carra, and D. Azoulay, "Managements of recurrent hepatocellular carcinoma after liver transplantation: a systematic review," World Journal of Gastroenterology, vol. 21, no. 39, pp. 11185-11198, 2015.

[25] V. A. Lingiah, M. Niazi, R. Olivo, F. Paterno, J. V. Guarrera, and N. T. Pyrsopoulos, "Liver transplantation beyond milan criteria," Journal of clinical and translational hepatology, vol. 8, no. 1, pp. 69-75, 2020.

[26] J. Asemota, M. Saleh, O. Igbinovia, and D. Burns, "A concise review on current trends in imaging and surgical management of hepatocellular carcinoma," Cureus, vol. 12, no. 7, Article ID e9191, 2020.

[27] Q. Ye, S. Ling, S. Zheng, and X. Xu, "Liquid biopsy in hepatocellular carcinoma: circulating tumor cells and circulating tumor DNA," Molecular Cancer, vol. 18, no. 1, p. 114, 2019.

[28] M. M. Ferreira, V. C. Ramani, and S. S. Jeffrey, "Circulating tumor cell technologies," Molecular Oncology, vol. 10, no. 3, pp. 374-394, 2016.

[29] G. Vona, A. Sabile, M. Louha et al., "Isolation by size of epithelial tumor cells," American Journal of Pathology, vol. 156, no. 1, pp. 57-63, 2000.

[30] E. Lin, T. Cao, S. Nagrath, and M. R. King, "Circulating tumor cells: diagnostic and therapeutic applications," Annual Review of Biomedical Engineering, vol. 20, no. 1, pp. 329-352, 2018.

[31] A. Agarwal, M. Balic, D. El-Ashry, and R. J. Cote, "Circulating tumor cells," The Cancer Journal, vol. 24, no. 2, pp. 70-77, 2018.

[32] N. A. Koonce, M. A. Juratli, C. Cai et al., "Real-time monitoring of circulating tumor cell (CTC) release after nanodrug or tumor radiotherapy using in vivo flow cytometry," Biochemical and Biophysical Research Communications, vol. 492, no. 3, pp. 507-512, 2017.

[33] Y. Suo, C. Xie, X. Zhu et al., "Proportion of circulating tumor cell clusters increases during cancer metastasis," Cytometry, Part A, vol. 91, no. 3, pp. 250-253, 2017.

[34] F. Castro-Giner and N. Aceto, "Tracking cancer progression: from circulating tumor cells to metastasis," Genome Medicine, vol. 12 , no. 1 , p. $31,2020$.

[35] P. Ramirez, L. Sáenz, P. A. Cascales-Campos et al., "Oncological evaluation by positron-emission tomography, circulating tumor cells and alpha fetoprotein in patients with hepatocellular carcinoma on the waiting list for liver transplantation," Transplantation Proceedings, vol. 48, no. 9, pp. 2962-2965, 2016.

[36] J. Zhou, Z. Zhang, H. Zhou et al., "Preoperative circulating tumor cells to predict microvascular invasion and dynamical detection indicate the prognosis of hepatocellular carcinoma," BMC Cancer, vol. 20, no. 1, p. 1047, 2020.

[37] J. DiNorcia, S. S. Florman, B. Haydel et al., "Pathologic response to pretransplant locoregional therapy is predictive of patient outcome after liver transplantation for hepatocellular carcinoma," Annals of Surgery, vol. 271, no. 4, pp. 616-624, 2020.

[38] M. Romero-Gutiérrez, M. Abanades Tercero, J. Ruiz Martín et al., "Characteristics and survival of hepatocellular carcinoma in non-cirrhotic liver," Revista Española de Enfermedades Digestivas, vol. 111, no. 10, pp. 760-766, 2019.
[39] C.-Y. Wang and S. Li, "Clinical characteristics and prognosis of 2887 patients with hepatocellular carcinoma," Medicine, vol. 98, no. 4, Article ID e14070, 2019.

[40] S. Wang, Y. Zheng, J. Liu, F. Huo, and J. Zhou, "Analysis of circulating tumor cells in patients with hepatocellular carcinoma recurrence following liver transplantation," Journal of Investigative Medicine: The Official Publication of the American Federation for Clinical Research, vol. 66, no. 5, pp. 1-6, 2018.

[41] Z. X. Zhu, J. W. Huang, M. H. Liao, and Y. Zeng, "Treatment strategy for hepatocellular carcinoma in China: radiofrequency ablation versus liver resection," Japanese Journal of Clinical Oncology, vol. 46, no. 12, pp. 1075-1080, 2016.

[42] S. Lee, T. W. Kang, K. D. Song et al., "Effect of microvascular invasion risk on early recurrence of hepatocellular carcinoma after surgery and radiofrequency ablation," Annals of Surgery, vol. 273, 2019.

[43] R. Inchingolo, A. Posa, M. Mariappan, and S. Spiliopoulos, "Locoregional treatments for hepatocellular carcinoma: current evidence and future directions," World Journal of Gastroenterology, vol. 25, no. 32, pp. 4614-4628, 2019.

[44] K. Yan, M. H. Chen, W. Yang et al., "Radiofrequency ablation of hepatocellular carcinoma: long-term outcome and prognostic factors," European Journal of Radiology, vol. 67, no. 2, pp. 336-347, 2008.

[45] EASL Clinical Practice Guidelines, "Management of hepatocellular carcinoma," Journal of Hepatology, vol. 69, no. 1, pp. 182-236, 2018. 